Research Article

\title{
Tuberculosis Test Usage and Medical Expenditures from Outpatient Insurance Claims Data, 2013
}

\author{
Kwame Owusu-Edusei Jr., Carla A. Winston, Suzanne M. Marks, \\ Adam J. Langer, and Roque Miramontes \\ Division of Tuberculosis Elimination, Centers for Disease Control and Prevention, Atlanta, GA, USA \\ Correspondence should be addressed to Kwame Owusu-Edusei Jr.; kfo0@cdc.gov
}

Received 14 April 2017; Revised 29 June 2017; Accepted 1 August 2017; Published 23 October 2017

Academic Editor: David C. Perlman

This is an open access article, free of all copyright, and may be freely reproduced, distributed, transmitted, modified, built upon, or otherwise used by anyone for any lawful purpose. The work is made available under the Creative Commons CC0 public domain dedication.

\begin{abstract}
Objective. To evaluate TB test usage and associated direct medical expenditures from 2013 private insurance claims data in the United States (US). Methods. We extracted outpatient claims for TB-specific and nonspecific tests from the 2013 MarketScan ${ }^{\circledR}$ commercial database. We estimated average expenditures (adjusted for claim and patient characteristics) using semilog regression analyses and compared them to the Centers for Medicare and Medicaid Services (CMS) national reimbursement limits. Results. Among the TB-specific tests, $1.4 \%$ of the enrollees had at least one claim, of which the tuberculin skin test was most common (86\%) and least expensive (\$9). The T-SPOT ${ }^{\circledR}$ was the most expensive among the TB-specific tests (\$106). Among nonspecific TB tests, the chest radiograph was the most used test $(78 \%)$, while chest computerized tomography was the most expensive (\$251). Adjusted average expenditures for the majority of tests $(\approx 74 \%)$ were above CMS limits. We estimated that total United States medical expenditures for the employer-based privately insured population for TB-specific tests were $\$ 53.0$ million in 2013 , of which enrollees paid $17 \%$ (\$9 million). Conclusions. We found substantial differences in TB test usage and expenditures. Additionally, employer-based private insurers and enrollees paid more than CMS limits for most TB tests.
\end{abstract}

\section{Introduction}

In spite of remarkable declines in tuberculosis (TB) incidence in the United States over the last six decades, TB continues to be a public health problem [1]. The major components of TB prevention and control efforts are timely identification of active TB cases, testing for TB infection among highrisk persons, and appropriate treatment of persons identified with $\mathrm{TB}$ disease or TB infection. In the United States, the majority $(77 \%)$ of TB prevention and control efforts (mainly, diagnostic and treatment services) are provided by the public sector through local health departments [2]. However, with the introduction of the Affordable Care Act, provision of health care services to diagnose and manage TB disease through the private sector, financed by private insurance, is likely to increase [2].

The purpose of this study was to examine TB test usage and associated medical expenditures by the private sector using outpatient insurance claims data available from the Truven Health MarketScan Commercial Claims Database. Estimates of private sector usage of individual TB tests will provide information on the relative contribution of the private sector in providing these services. Estimates of the average expenditures on each test are needed for budgeting and cost projections for future testing and diagnosis programs. They are also needed for estimating the overall cost of $\mathrm{TB}$ prevention and control activities. Additionally, they are indispensable inputs for conducting economic evaluations-such as cost-effectiveness and cost-benefit analysis-of TB testing and prevention programs. Comparisons of private versus public insurance expenditures on TB tests provide measures of price differentials that can help to determine the economic cost of testing. Finally, results from this study will provide TB test usage and medical expenditure benchmarks that can be useful for assessing trends. 


\section{Materials and Methods}

We used retrospective data on private insurance claims for reimbursement (henceforth referred to as "claims") from the Truven Health MarketScan Commercial Claims Database for 2013. The MarketScan database includes health insurance claims across the continuum of health care (including inpatient, outpatient, and outpatient pharmacy) and enrollment data from large employers and health insurance plans across the United States [3]. The 2013 data contained claims information on more than 43 million people including employees, their spouses, and dependents (Truven Health MarketScan Commercial Claims Database), representing $\approx 25 \%$ of the estimated employer-based privately insured population in the United States [4].

We compiled a list of current procedural terminology (CPT) codes for testing methods used to screen for TB infection (tuberculin skin tests [TSTs] and interferon gamma release assays [IGRAs]) as well as tests for active TB disease diagnoses (chest radiograph, chest computerized tomography, sputum smear and culture, nucleic acid amplification, and drug susceptibility testing). These codes were drawn from various sources, including the American Medical Association (AMA) and Centers for Medicare and Medicaid Services (CMS) websites [5, 6]. We categorized CPT codes as representing TB-specific (the target pathogenic species was Mycobacterium tuberculosis only) or nonspecific (the target pathogenic species included-or could potentially include-Mycobacterium tuberculosis) tests. The list of CPT codes and their description according to AMA [5] is presented in Table 1. We used the brand names for the respective IGRA testing method CPT codes (i.e., QuantiFERON ${ }^{\circledR}-T B$ Gold In-Tube [QFT-GIT] for 86480 and T-SPOT.TB [T-Spot] for 86481 ) because they were the only IGRA tests approved by the Food and Drug Administration (FDA) as of 2013 [7].

Using the compiled CPT codes, we extracted claims for tests performed on enrollees in 2013 from the outpatient table. We computed claims rate (number of claims per 100,000 enrollees) as the measure of usage for each CPT code using enrollee data from the enrollment table as the denominator. The associated medical expenditures data for each test were the total payment, which included payments by both enrollee and insurance plan, provided with each claim record [3]. As a result, unless otherwise specified, all the expenditures reported (for each test and overall total) in this study are the total payments from insurance plans and enrollees. Next, following previously published methods $[8,9]$, we deleted claims with payments $\leq \$ 1$ and determined the average medical expenditures for each test (CPT code)-referred to as the unadjusted average medical expenditures. Deleting claims with payments $\leq \$ 1$ was necessary given that some encounters contained $\leq \$ 1$ total payments for capitated services that might not represent the correct reimbursements [3]. The claim records with $\leq \$ 1$ represented $<6 \%$ of the overall total. Given the potential for outliers to influence the estimated unadjusted average medical expenditures, we reestimated the average medical expenditures by using a semilog regression that controlled for claim and patient characteristics-referred to as the adjusted average medical expenditures.
In the semilog regression analyses, the dependent variables were the natural logs of medical expenditures (to reduce the influence of outliers) for each test and the independent variables were age, age-squared variable, quantity of services [number of services performed for a claim], gender [male/female], data supplier [employer/health plan], drug benefit [whether or not enrollee's insurance plan included prescription drug coverage], region of the United States [South/Northeast/North/Central/West/unknown], and health plan type [comprehensive/exclusive provider organization (EPO)/health maintenance organization (HMO)/point of service (POS)/preferred provider organization (PPO)/ consumer driven health plan (CDHP)/high deductible health plan (HDHP)] based on previous studies [8-12]. For easy interpretation of the regression results, the coefficients from the regression analyses were transformed as coefficient $(\beta) * 100$ and interpreted as the relative change (in percentage) per unit change in the independent variable (IV) for the continuous IVs and as $\left(e^{\beta}-1\right) * 100$ and interpreted as the relative difference (in percentage) in the estimated medical expenditures when compared with the referent category [13].

We examined differences in usage among the TB-specific and nonspecific tests by using 2-sided $z$ test for differences in claims rates and used the regression analysis to test for differences in average medical expenditures between tests by including them as categorical variables (equal to 1,0 otherwise). The adjusted average medical expenditures for each CPT were compared to the CMS fee schedule national limit for 2013 [6] and presented as relative difference in percentages. We also compared the adjusted estimates among the three commonly used TB-specific tests-86480 (QFTGIT), 86481 (T-Spot), and 86580 (TST). We determined the number of enrollees who had at least one claim for the TBspecific tests and estimated the proportion tested for TB. Finally, we used straight-line extrapolation to estimate the overall total medical expenditures of the TB-specific tests for the entire United States population who had employerbased health insurance in 2013. Because of the potential for overestimation of costs attributable to $\mathrm{TB}$, we did not include the overall total medical expenditures for the nonspecific TB tests. We also estimated the overall total medical expenditures for the three commonly used TB-specific tests-86480 (QFTGIT), 86481 (T-Spot), and 86580 (TST).

We used MEDSTAT DataProbe version 3.3.15 (Truven Health Analytics Inc., Ann Arbor, MI) for data extraction. Microsoft Excel, version 2013 (Microsoft Corporation, Redmond, WA), was used for summary results and graphic presentations and STATA version 14.0 (StataCorp LP, College Station, TX) was used for all regression analyses including result validation and diagnostics.

\section{Results}

3.1. TB-Specific Test Usage. The estimated claims rates for the TB-specific tests are presented in Figure 1. Based on the data from the 2013 outpatient table, the estimated claims rates ranged from $0.05 / 100,000$ (87557 [nucleic acid, quantitative (NAQ); Mycobacteria tuberculosis]) to $1,323 / 100,000$ (86580 [TST]) (Figure 1). Overall, TSTs made up $86.2 \%$ of the 
TABLE 1: Current procedural terminology (CPT) codes and their description for the TB-specific and the nonspecific TB tests.

\begin{tabular}{|c|c|}
\hline CPT code & Description (source: American Medical Association online CPT search [5]) \\
\hline \multicolumn{2}{|r|}{ TB-specific tests } \\
\hline 86480 & $\begin{array}{l}\text { Tuberculosis test, cell mediated immunity antigen response measurement; gamma interferon (QuantiFERON-TB Gold } \\
\text { In-Tube [QFT-GIT] })^{\dagger}\end{array}$ \\
\hline 86481 & $\begin{array}{l}\text { Tuberculosis test, cell mediated immunity antigen response measurement; enumeration of gamma interferon-producing } \\
\text { T-cells in cell suspension (T-SPOT.TB [T-Spot] })^{\dagger}\end{array}$ \\
\hline 86580 & Skin test; tuberculosis, intradermal \\
\hline 87555 & Infectious agent detection by nucleic acid (DNA or RNA); Mycobacterium tuberculosis, direct probe technique \\
\hline 87556 & Infectious agent detection by nucleic acid (DNA or RNA); Mycobacterium tuberculosis, amplified probe technique \\
\hline 87557 & Infectious agent detection by nucleic acid (DNA or RNA); Mycobacterium tuberculosis, quantification \\
\hline \multicolumn{2}{|r|}{ Nonspecific TB tests } \\
\hline 71020 & Radiologic examination, chest, 2 views, frontal and lateral \\
\hline 71260 & Computed tomography, thorax; with contrast material(s) \\
\hline 87116 & $\begin{array}{l}\text { Culture, tubercle, or other acid-fast bacilli (e.g., TB, AFB, and mycobacteria) any source, with isolation and presumptive } \\
\text { identification of isolates }\end{array}$ \\
\hline 87118 & Culture, mycobacterial, definitive identification, each isolate \\
\hline 87143 & Culture, typing; gas liquid chromatography (GLC) or high pressure liquid chromatography (HPLC) method \\
\hline 87149 & $\begin{array}{l}\text { Culture, typing; identification by nucleic acid (DNA or RNA) probe, direct probe technique, per culture or isolate, each } \\
\text { organism probed }\end{array}$ \\
\hline 87150 & $\begin{array}{l}\text { Culture, typing; identification by nucleic acid (DNA or RNA) probe, amplified probe technique, per culture or isolate, } \\
\text { each organism probed }\end{array}$ \\
\hline 87153 & Culture, typing; identification by nucleic acid sequencing method, each isolate (e.g., sequencing of the $16 \mathrm{~S}$ rRNA gene) \\
\hline 87158 & Culture, typing; other methods \\
\hline 87188 & Susceptibility studies, antimicrobial agent; macrobroth dilution method, each agent \\
\hline 87190 & Susceptibility studies, antimicrobial agent; mycobacteria, proportion method, each agent \\
\hline 87206 & $\begin{array}{l}\text { Smear, primary source with interpretation; fluorescent and/or acid fast stain for bacteria, fungi, parasites, viruses, or cell } \\
\text { types }\end{array}$ \\
\hline 87550 & Infectious agent detection by nucleic acid (DNA or RNA); mycobacteria species, direct probe technique \\
\hline 87551 & Infectious agent detection by nucleic acid (DNA or RNA); mycobacteria species, amplified probe technique \\
\hline 87552 & Infectious agent detection by nucleic acid (DNA or RNA); mycobacteria species, quantification \\
\hline 87798 & $\begin{array}{l}\text { Infectious agent detection by nucleic acid (DNA or RNA), not otherwise specified; amplified probe technique, each } \\
\text { organism }\end{array}$ \\
\hline 87799 & Infectious agent detection by nucleic acid (DNA or RNA), not otherwise specified; quantification, each organism \\
\hline
\end{tabular}

${ }^{\dagger}$ The brand names for the respective IGRA testing method CPT codes (i.e., QuantiFERON-TB Gold In-Tube [QFT-GIT] for 86480 and T-SPOT.TB [T-Spot] for 86481) were used because they were the only IGRA tests approved by the Food and Drug Administration (FDA) as of 2013 [7].

TB-specific tests and similarly $86.3 \%$ among the three commonly used TB-specific tests (QFT-GIT, T-Spot, and TST). The claims rate for 87557 (NAQ; Mycobacteria tuberculosis) was significantly $(p<0.01)$ lower than the claims rates for all the other TB-specific tests. Conversely, the claims rate for 86580 (TST) was significantly $(p<0.01)$ higher than the claims rates for all the other TB-specific tests (Figure 1). We found that 609,394 enrollees had at least one claim for TB-specific tests, representing $1.4 \%$ of the total number of enrollees in 2013.

3.2. Nonspecific TB Test Usage. The estimated claims rates for the nonspecific TB tests are presented in Figure 2. Among the nonspecific TB tests, the estimated claims rates ranged from 0.05/100,000 (87552 [nucleic acid, quantitative (NAQ); mycobacteria species]) to $10,315 / 100,000$ (71020 [chest radiograph; 2 views]) (Figure 2). Overall, 71020 (chest radiograph;
2 views) made up over $78 \%$ of the nonspecific TB tests. The estimated claims rate for upstream tests in the diagnostic algorithm (such as 71020 [chest radiograph; 2 views]) was significantly higher than the claims rates of tests that might be expected to occur later in a diagnostic process (such as 87550 [nucleic acid direct probe (NADP); mycobacteria species] and 87552 [NAQ; mycobacteria species] $)(p<0.01)$ (Figure 2).

3.3. Medical Expenditures. Table 2 shows the full regression results used for estimating the adjusted average medical expenditures for the TB-specific tests with the highest usage (86580 [TST]), which included a binary variable to allow for evaluation of the difference in medical expenditures between 86580 (TST) and 86480 (QFT-GIT). Our regression results indicated a quadratic relationship between medical expenditures for the test and age, namely, increased average 
TB-specific test claims rate, 2013

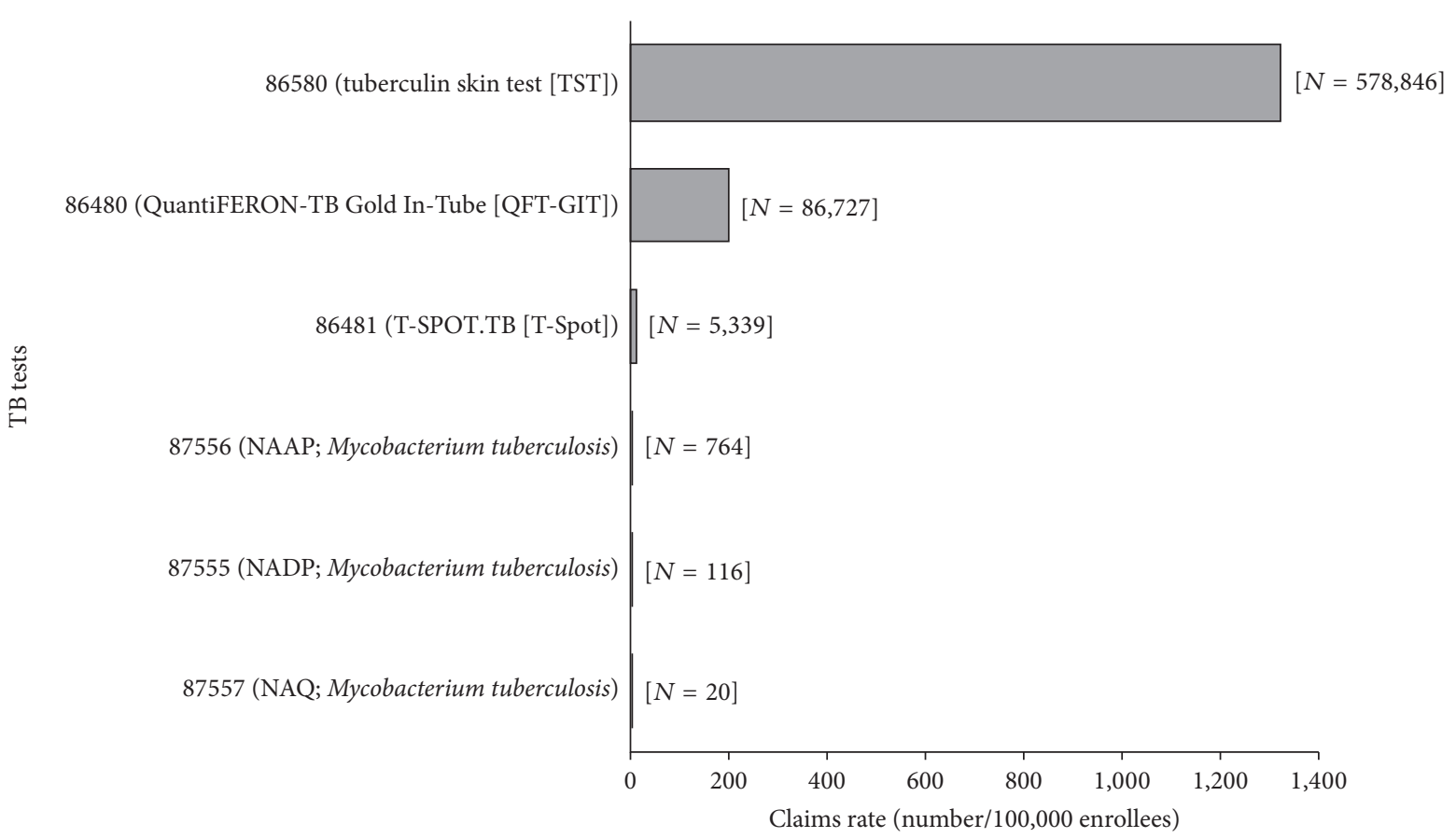

FIGURE 1: Estimated claims rates (number/100,000 enrollees) for the TB-specific tests. TB, tuberculosis; NAAP, nucleic acid amplification probe; NADP, nucleic acid direct probe; NAQ, nucleic acid quantification. The brand names for the respective IGRA testing method CPT codes (i.e., QuantiFERON-TB Gold In-Tube [QFT-GIT] for 86480 and T-SPOT.TB [T-Spot] for 86481) were used because they were the only IGRA tests approved by the Food and Drug Administration (FDA) as of 2013 [7].

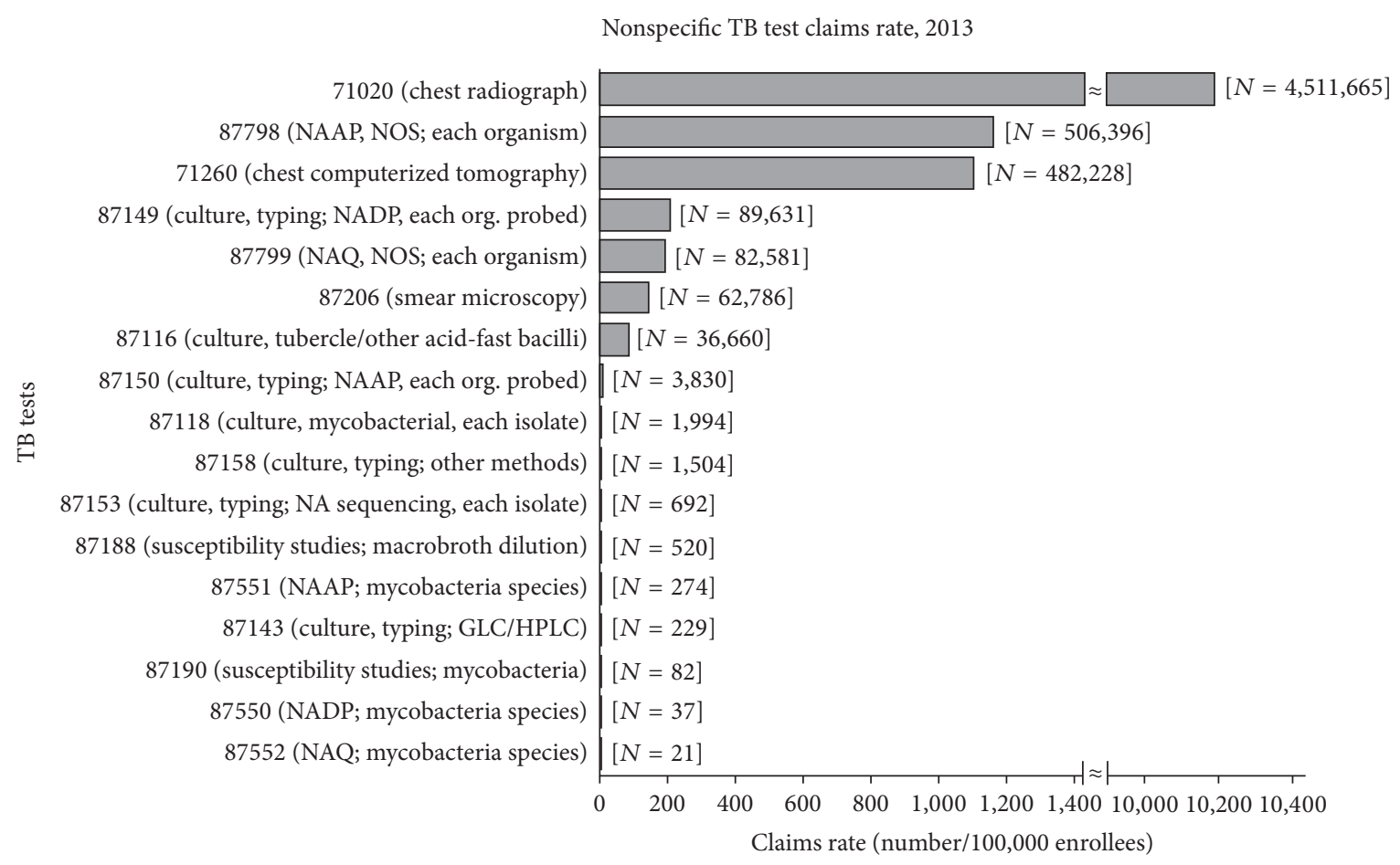

FIGURE 2: Estimated claims rates (number/100,000 enrollees) for the nonspecific TB tests. TB, tuberculosis; NAAP, nucleic acid amplification probe; NADP, nucleic acid direct probe; NAQ, nucleic acid quantification; NA, nucleic acid; NOS, not otherwise specified; GLC, gas liquid chromatography; HPLC, high pressure liquid chromatography. 
TABLE 2: Summary regression results. Dependent variable: log of the medical expenditures of test for 86580 (TST) and 86480 (QFT-GIT). $N=628,595$.

\begin{tabular}{|c|c|c|}
\hline Independent variables & Coefficient $(\beta)^{\dagger}[95 \% \mathrm{CI}]$ & Transformed coefficient (\%) $[95 \% \mathrm{CI}]^{\ddagger}$ \\
\hline Age of patient & $0.0048[0.0045 \text { to } 0.0051]^{* * *}$ & $0.48[0.45$ to 0.51$]$ \\
\hline Age-squared variable & $-0.0000558[-0.0000603 \text { to }-0.0000513]^{* * *}$ & $-0.0056[-0.0060$ to -0.0051$]$ \\
\hline Quantity of services & $0.16263[0.1577 \text { to } 0.1676]^{* * *}$ & $16.26[15.77$ to 16.76$]$ \\
\hline \multicolumn{3}{|l|}{ Gender } \\
\hline Male & Referent & Referent \\
\hline Female & $-0.0032[-0.0059 \text { to }-0.0006]^{* *}$ & $-0.32[-0.59$ to -0.06$]$ \\
\hline \multicolumn{3}{|l|}{ Data supplier } \\
\hline Employer & Referent & Referent \\
\hline Health plan & $-0.0059[-0.0091 \text { to }-0.0026]^{* * *}$ & $-0.59[-0.91$ to -0.26$]$ \\
\hline \multicolumn{3}{|l|}{ Drug coverage? } \\
\hline No & Referent & Referent \\
\hline Yes & $-0.005[-0.0086 \text { to }-0.0014]^{* * *}$ & $-0.5[-0.86$ to -0.14$]$ \\
\hline \multicolumn{3}{|l|}{ Region } \\
\hline South & Referent & Referent \\
\hline Northeast & $0.1147[0.1111 \text { to } 0.1183]^{* * *}$ & $12.15[11.75$ to 12.56$]$ \\
\hline North Central & $0.2134[0.2092 \text { to } 0.2176]^{* * *}$ & $23.79[23.27$ to 24.31$]$ \\
\hline West & $0.2795[0.2757 \text { to } 0.2833]^{* * *}$ & $32.25[31.75$ to 32.75$]$ \\
\hline Unknown & $0.1743[0.1653 \text { to } 0.1833]^{* * *}$ & $19.04[17.97$ to 20.12$]$ \\
\hline \multicolumn{3}{|l|}{ Health plan type } \\
\hline Comprehensive & Referent & Referent \\
\hline Exclusive provider organization (EPO) & $-0.0692[-0.083 \text { to }-0.0553]^{* * *}$ & $-6.69[-7.96$ to -5.38$]$ \\
\hline Health maintenance organization (HMO) & $-0.007[-0.0195$ to 0.0056$]$ & $-0.7[-1.93$ to 0.56$]$ \\
\hline Point of service (POS) & $-0.0478[-0.0609 \text { to }-0.0348]^{* * *}$ & $-4.67[-5.91$ to -3.42$]$ \\
\hline Preferred provider organization (PPO) & $-0.0306[-0.0428 \text { to }-0.0183]^{* * *}$ & $-3.01[-4.19$ to -1.81$]$ \\
\hline POS with capitation & $-0.1314[-0.1502 \text { to }-0.1127]^{* * *}$ & $-12.31[-13.95$ to -10.66$]$ \\
\hline Consumer driven health plan (CDHP) & $-0.0795[-0.0928 \text { to }-0.0662]^{* * *}$ & $-7.64[-8.86$ to -6.41$]$ \\
\hline High deductible health plan (HDHP) & $-0.0996[-0.1129 \text { to }-0.0863]^{* * *}$ & $-9.48[-10.68$ to -8.27$]$ \\
\hline \multicolumn{3}{|l|}{ Expenditures (difference) } \\
\hline 86580 (TST) & Referent & Referent \\
\hline 86480 (QFT-GIT) & $2.0627[2.0587 \text { to } 2.0666]^{* * *}$ & $686.72[683.58$ to 689.79$]$ \\
\hline
\end{tabular}

Significance levels: ${ }^{* * *} p<0.01 ;{ }^{* *} p<0.05 .{ }^{\dagger}$ The $t$-values associated with the coefficients were derived from bootstrap-generated standard errors with 50 replications. ${ }^{*}$ Because the dependent variables in the regression analyses were the natural log of dependent variable (medical expenditures), the coefficients were transformed as coefficient $(\beta) * 100$ and interpreted as the relative change (in percentage) per unit change in the independent variable (IV) for the continuous IVs and as $\left(e^{\beta}-1\right) * 100$ and interpreted as the relative difference (in percentage) in the estimated medical expenditures when compared with the referent (or omitted) category [13]. The brand names for the respective IGRA testing method CPT codes (i.e., QuantiFERON-TB Gold In-Tube [QFT-GIT] for 86480 and T-SPOT.TB [T-Spot] for 86481) were used because they were the only IGRA tests approved by the Food and Drug Administration (FDA) as of 2013 [7].

expenditures by age at a decreasing rate by age (Table 2 ). In addition, we found that a unit increase in the number of services for a claim resulted in a $16.3 \%(p<0.01)$ increase in the associated average medical expenditures for the test. Female enrollees' average medical expenditures were $0.3 \%$ $(p<0.05)$ lower than those for males. Average medical expenditures from health plans were $0.6 \%(p<0.01)$ lower than those from self-insured employers, and, except for the HMO plan type, all the other health plan types had higher $(p<0.01)$ medical expenditures, on average. Medical expenditures from the South were 12\%-32\% $(p<$ $0.01)$ lower than those from the other regions of the United States. Finally, the coefficient $(\beta)$ on the 86480 (QFT-GIT) categorical variable implied that the medical expenditures for 86480 (QFT-GIT) were $\approx 7$ times (i.e., $\left(e^{\beta}-1\right) * 100$; $p<0.01$ ) higher than that for 86580 (TST) on average (Table 2).

Because of the large number of regression analyses conducted to estimate and to compare the medical expenditures for the 23 tests, we are not able to show full results for the other tests-they are available from the lead author. Except for 87552 (nucleic acid, quantitative (NAQ); mycobacteria species), which had a substantially low number of observations $(n=21)$, the qualitative and quantitative results of the coefficients were largely consistent across all the regression results obtained from the other tests.

3.4. Medical Expenditures for TB-Specific Tests. The summary results for the estimated adjusted and unadjusted average medical expenditures for the TB-specific tests are presented 
TABLE 3: Summary results showing the unadjusted and adjusted average medical expenditures for all the tests (2013 United States dollars).

\begin{tabular}{|c|c|c|c|c|}
\hline Test & $\begin{array}{c}\text { Unadjusted average } \\
\text { medical expenditures } \\
{[\mathrm{SD}]}\end{array}$ & $\begin{array}{c}\text { Adjusted average medical } \\
\text { expenditures }{ }^{\dagger} \\
{[95 \% \mathrm{CI}]}\end{array}$ & $\begin{array}{l}\text { CMS } \\
\text { limit }\end{array}$ & $\begin{array}{c}\text { Relative } \\
\text { difference from } \\
\text { CMS } \\
\end{array}$ \\
\hline \multicolumn{5}{|c|}{ TB-specific tests } \\
\hline 86481 (T-SPOT.TB [T-Spot]) & $140.77[104.52]$ & $105.81[103.72-107.94]^{* *}$ & 102.99 & $2.7 \%$ \\
\hline 87556 (NAAP; Mycobacterium tuberculosis) & $123.34[141.54]$ & $74.45[68.53-80.87]$ & 48.24 & $54.3 \%$ \\
\hline 87557 (NAQ; Mycobacterium tuberculosis) & $77.53[25.84]$ & $73.1[68.09-78.47]$ & 58.88 & $24.1 \%$ \\
\hline 86480 (QuantiFERON-TB Gold In-Tube [QFT-GIT]) & $86.96[88.08]$ & $69.14[68.83-69.46]^{* *}$ & 85.20 & $-18.8 \%$ \\
\hline 87555 (NADP; Mycobacterium tuberculosis) & $73.77[75.4]$ & $45.78[37.51-55.88]^{* *}$ & 27.57 & $66.1 \%$ \\
\hline 86580 (tuberculin skin test [TST]) & $10.52[26.59]$ & $8.68[8.67-8.69]$ & 8.64 & $0.4 \%$ \\
\hline \multicolumn{5}{|c|}{ Nonspecific TB tests } \\
\hline 71260 (chest computerized tomography) & $454.23[573.78]$ & $251.11[250.33-251.89]^{* *}$ & 266.40 & $-5.7 \%$ \\
\hline 87153 (culture, typing; NA sequencing, each isolate) & $193.31[149.87]$ & $145.08[135.66-155.15]$ & 158.57 & $-8.5 \%$ \\
\hline 87150 (culture, typing; NAAP, each org. probed) & $275.85[396.52]$ & $135.04[130.26-140]^{* *}$ & 48.24 & $179.9 \%$ \\
\hline 87552 (NAQ; mycobacteria species) & $127.85[88.91]$ & $92.66[52.48-163.61]$ & 58.88 & $57.4 \%$ \\
\hline 87799 (NAQ, NOS; each organism) & $122.78[307.61]$ & $78.86[78.36-79.36]$ & 58.88 & $33.9 \%$ \\
\hline 87550 (NADP; mycobacteria species) & $84.55[85.42]$ & $60.42[45.03-81.07]$ & 27.57 & $119.1 \%$ \\
\hline 87798 (NAAP, NOS; each organism) & $78.58[138.91]$ & $47.54[47.44-47.63]$ & 48.24 & $-1.4 \%$ \\
\hline 87551 (NAAP; mycobacteria species) & $84.12[221.03]$ & $45.89[41.94-50.21]^{*}$ & 48.28 & $-5.0 \%$ \\
\hline 87188 (susceptibility studies; macrobroth dilution) & $125.81[207.41]$ & $39.65[35.72-44.01]^{* *}$ & 9.12 & $334.7 \%$ \\
\hline 71020 (chest radiograph; 2 views) & $63.39[101.58]$ & $38.16[38.13-38.2]$ & 30.96 & $23.3 \%$ \\
\hline 87143 (culture, typing; GLC/HPLC) & $60.96[61.75]$ & $37.08[32.77-41.96]^{*}$ & 17.22 & $115.3 \%$ \\
\hline 87116 (culture, tubercle/other acid-fast bacilli) & $57.36[91.95]$ & $31.32[30.94-31.71]^{* *}$ & 14.85 & $110.9 \%$ \\
\hline 87190 (susceptibility studies; mycobacteria) & $51.69[70.78]$ & $24.8[18.56-33.13]$ & 7.77 & $219.1 \%$ \\
\hline 87118 (culture, mycobacterial, each isolate) & $37.62[50.52]$ & $20.35[19.38-21.37]^{* *}$ & 15.04 & $35.3 \%$ \\
\hline 87149 (culture, typing; NADP, each org. probed) & $25.35[75.55]$ & $18.51[18.44-18.57]^{* *}$ & 27.57 & $-32.9 \%$ \\
\hline 87206 (smear microscopy) & $25[52.12]$ & $12.69[12.58-12.8]^{* *}$ & 7.39 & $72.4 \%$ \\
\hline 87158 (culture, typing; other methods) & $14.53[28.34]$ & 9.08 [8.7-9.48] & 7.19 & $26.3 \%$ \\
\hline
\end{tabular}

${ }^{\dagger}$ Arranged in decreasing order of magnitude. Significance levels: ${ }^{* *} p<0.01,{ }^{*} p<0.05$ higher than the next estimate. CMS, Centers for Medicare and Medicaid Services; SD, standard deviation; TB, tuberculosis; NAAP, nucleic acid amplification probe; NADP, nucleic acid direct probe; NAQ, nucleic acid quantification; NA, nucleic acid; NOS, not otherwise specified; GLC, gas liquid chromatography; HPLC, high pressure liquid chromatography. The brand names for the respective IGRA testing method CPT codes (i.e., QuantiFERON-TB Gold In-Tube [QFT-GIT] for 86480 and T-SPOT.TB [T-Spot] for 86481) were used because they were the only IGRA tests approved by the Food and Drug Administration (FDA) as of 2013 [7].

in the upper panel of Table 3. The estimated adjusted average medical expenditures ranged from $\$ 8.68$ (86580 [TST]) to $\$ 105.81$ (86481 [T-Spot]). The average medical expenditures for 86580 (TST) were significantly lower $(p<0.01)$ than the average medical expenditures for all the other TBspecific tests, while the average medical expenditures for 86481 (T-Spot) were significantly higher $(p<0.01)$ than the average medical expenditures of all the other TB-specific tests. When compared to their respective CMS national limits, the adjusted medical expenditures were generally higher-ranging from $0.4 \%$ for 86580 (TST) to $66.1 \%$ higher for 87555 (nucleic acid direct probe (NADP); Mycobacterium tuberculosis). The exception was 86480 (QFT-GIT) which was $18.8 \%$ lower than the CMS national limit (Table 3).

Based on the volume of all the TB-specific tests and their associated medical expenditures, we estimated that the overall total medical expenditures for TB-specific tests performed for these enrollees in 2013 were \$13.7 million (\$13.6 million when focusing on the three commonly used
TB-specific tests-QFT-GIT, T-Spot, and TST). Extrapolating to the entire employer-based privately insured population (i.e., 169.0 million [4]) in the United States, we estimated that the total medical expenditures among the employer-based privately insured population for all the TB-specific tests were $\$ 53.0$ million in 2013 (\$52.6 million for the three commonly used TB-specific tests-QFT-GIT and T-Spot [\$30.1 million] and TST [\$22.5 million]). Enrollees paid approximately $17 \%$ (\$9 million) of the overall total medical expenditures for the TB-specific tests.

3.5. Average Medical Expenditures for Nonspecific TB Tests. The summary results for the estimated adjusted and unadjusted average medical expenditures for the nonspecific TB tests are presented in the lower panel of Table 3. The estimated adjusted average medical expenditures ranged from $\$ 9.08$ (87158 [culture, typing; other methods]) to $\$ 251.11$ (71260 [chest computerized tomography]). The adjusted average medical expenditures for 87158 (culture, typing; other 
methods) were significantly $(p<0.01)$ lower than the medical expenditures for all the other nonspecific TB tests, while the estimated adjusted average medical expenditures for 71260 (chest computerized tomography) were significantly $(p<0.01)$ higher than the average medical expenditures for all the other nonspecific TB tests. Most of the estimated adjusted average medical expenditures for the nonspecific TB tests were higher than the CMS national limits-12 of the 17 nonspecific TB tests (Table 3 ). The largest difference was the estimated average medical expenditures for 87188 (susceptibility studies; macrobroth dilution), which were $334.7 \%$ higher than the CMS national limit. On the other hand, the average medical expenditures of 87149 (culture, typing; NADP, each org. probed) were $32.9 \%$ lower than the CMS national limits (Table 3 ).

\section{Discussion}

The results from our analysis of TB-associated CPT codes from 2013 health insurance outpatient claims data indicated substantial differences in TB test usage and the associated medical expenditures. Among the TB-specific tests, TST was the most used (86\%) and least expensive (\$9). The most expensive TB-specific test was the T-Spot test $(\approx \$ 106)$, which is consistent with a study from the United Kingdom [14], although they found that T-Spot was less expensive per active TB case averted relative to QFT-GIT. Among the nonspecific TB tests, chest radiograph was the most used test $(78 \%)$, while chest computerized tomography was the most expensive $(\approx \$ 251)$.

The TST has been the standard TB infection test in most parts of the world for over a century [15-17]. Although IGRAs were introduced as alternatives to the TST for the diagnosis of TB infection in the United States over a decade ago $[18,19]$, our results suggest that TST remains the most widely used test performed among the privately insured population in 2013. In fact, the only test that was used more than TST was the chest radiograph, which is a nonspecific TB test because it is used for the diagnosis/evaluation of a myriad of other conditions including-but not limited to-pneumonia, cardiac conditions, bone abnormalities, and chest wall conditions [20]. In addition, chest radiographs are used not only for investigating the presence of active disease among people with clinical signs, but also for ruling out active disease among asymptomatic people with a current or past record of a positive test for TB infection.

We found that the majority of the estimated medical expenditures were higher than their CMS national reimbursement limits. Five out of the six TB-specific tests were $0.4 \%-66.1 \%$ higher, although the adjusted average medical expenditures of the QFT-GIT test were almost one-fifth lower than the CMS limit. Based on the estimated volume and the associated medical expenditures, we estimated that the total medical expenditures of all the TB-specific tests performed among the entire United States employer-based privately insured population in 2013 were $\$ 53.0$ million, although almost all (99\%) of the estimated total medical expenditures were from three tests-QFT-GIT, T-Spot, and TST. Additionally, although the IGRAs were used far less often than the
TSTs (13.7\% versus $86.3 \%)$ among the three commonly used TB-specific tests (QFT-GIT, T-Spot, and TST), they made up the majority of the overall total expenditures (57\%).

\section{Limitations and Strengths}

There are some limitations with the use of administrative claims data for this analysis. The database contains information on select employers/health plans based on their willingness to participate. The commercial claims data includes information on persons aged 0-64 years and the proportion of enrollees who were coded as female in 2013 was similar to the United States general population (51\% [21]). However, there were a slightly lower proportion of enrollees from the Southern region than the general United States general population (34\% versus $37.5 \%$ [21]), although approximately $3 \%$ of the enrollees' regions were coded as missing/unknown and the proportions for the other regions were similar to the United States general population. Because the data includes information predominantly on large employers [22] in large cities, a relatively higher proportion of the enrollees were from urban areas. As a result, the data were a convenience sample that may not be generalizable to the entire employerbased privately insured population in the United States. Additionally, information on race/ethnicity and foreign-born status is not available. Thus, our extrapolated estimate of the total medical expenditures for all the TB-specific tests among the entire United States employer-based privately insured population in 2013 is a crude estimate and should be interpreted with caution. Also, as with any administrative database, there may be inaccurate information for one or a combination of reasons, including clinician, data unavailability, or data entry errors $[23,24]$. As an example, the region variable (geographic location of primary beneficiary's residence) was coded as missing or unknown in some of the claim records (see Table 2), although for a relatively small proportion of the overall total number of claim records we extracted-3\%. We did not include CPT codes for magnetic resonance imaging (MRI) which is sometimes used for TB diagnosis or treatment evaluation [25]. Our analyses do not account for the fact that multiple CPTs codes may pertain to the same individual in the course of testing for TB infection or ruling in or out $\mathrm{TB}$ disease diagnosis, nor do we account for the cost of clinic visits or patient opportunity costs in obtaining testing.

The current study has several strengths. First, although the enrollees in the database may not be representative of the employer-based privately insured population, it is one of the largest private insurance databases in the United States. With data on over 43 million enrollees in 2013, the database contains information on $\approx 25 \%$ of the estimated United States population with employer-based private insurance in 2013 (169.0 million people [3]). Secondly, the medical expenditures information provided in the database is payments (not charges), so our estimates represent actual dollar amounts paid for the tests performed for these enrollees in 2013 [3,22]. Finally, this study adds to the literature by providing relevant private sector perspective on TB test usage and the associated medical expenditures in the United States. 


\section{Conclusion}

Our study found substantial differences in TB test usage as well as the associated medical expenditures based on the 2013 data. The information on the unique TB test usage and medical expenditures provides the benchmarks necessary for monitoring private sector testing practices and medical expenditures over time, especially with increased access to private health insurance through the Affordable Health Care Act beginning in 2014. After examining trends in the use of IGRAs and TST among privately insured persons in the United States over a 15-year period (2000-2014), a recent study suggested a gradual shift from the use of TST to IGRAs [26]. Future studies can examine the trends in the individual test payments, including the associated shift, if any, in the total expenditures from TST to IGRAs over time.

\section{Ethical Approval}

No institutional review board (IRB) approval was required because only secondary data were used.

\section{Disclosure}

The findings and conclusions in this report are those of the authors and do not necessarily represent the official position of the United States Centers for Disease Control and Prevention (CDC). Mention of company names or products does not imply endorsement by CDC.

\section{Conflicts of Interest}

The authors declare that they have no conflicts of interest.

\section{Acknowledgments}

The authors thank Dr. Samuel Shillcutt for his critical review of earlier drafts of the manuscript.

\section{References}

[1] Centers for Disease Control and Prevention, "Reported Tuberculosis in the United States 2014," Atlanta, Ga, U.S.A Department of Health and Human Services, 2015.

[2] V. Balaban, S. M. Marks, S. C. Etkind et al., "Tuberculosis elimination efforts in the United States in the era of insurance expansion and the affordable care act," Public Health Reports, vol. 130, no. 4, pp. 349-354, 2015.

[3] Truven Health Analytics Inc, "Marketscan Research Database: User Guide and Database Dictionary. Ann Arbor, MI," 2013.

[4] J. C. Smith and C. Medalia, "Health insurance coverage in the United States," Census Bureau P60-250, 2014, Washington, DC: Current Population Reports.

[5] American Medical Association, "CPT Code/Relative Value Search Chicago, IL: American Medical Association,” 2015, https: //ocm.ama-assn.org/OCM/CPTRelativeValueSearch.do?submitbutton=accept.

[6] Centers for Medicare \& Medicaid Services, "Clinical Diagnostic Laboratory Fee Schedule 2013,” 2014, https://www.cms.gov/ Medicare/Medicare-Fee-for-Service-Payment/PhysicianFeeSched/ index.html? redirect=/physicianFeeSched.
[7] Centers for Disease Control and Prevention, "Fact Sheets: Interferon-Gamma Release Assays (IGRAs) - Blood Tests for TB Infection Atlanta, GA," Centers for Disease Control and Prevention, 2012, http://www.cdc.gov/tb/publications/factsheets/ testing/igra.htm.

[8] K. Owusu-Edusei, E. W. Flagg, and T. L. Gift, "Hospitalization cost per case of neonatal herpes simplex virus infection from claims data," Journal of Pediatric Nursing, vol. 30, no. 2, pp. 346352, 2015.

[9] K. Owusu-Edusei, C. E. Introcaso, and H. W. Chesson, "Hospitalization cost of congenital syphilis diagnosis from insurance claims data in the United States," Sexually Transmitted Diseases, vol. 40, no. 3, pp. 226-229, 2013.

[10] S. S. Shrestha, P. Zhang, L. Barker, and G. Imperatore, "Medical expenditures associated with diabetes acute complications in privately insured U.S. youth," Diabetes Care, vol. 33, no. 12, pp. 2617-2622, 2010.

[11] S. S. Shrestha, P. Zhang, A. Albright, and G. Imperatore, "Medical expenditures associated with diabetes among privately insured U.S. youth in 2007," Diabetes Care, vol. 34, no. 5, pp. 1097-1101, 2011.

[12] K. Owusu-Edusei, S. M. Marks, R. Miramontes, E. L. Stockbridge, and C. A. Winston, "Tuberculosis hospitalization expenditures per patient from private health insurance claims data, 2010-2014," International Journal of Tuberculosis and Lung Disease, vol. 21, no. 4, pp. 398-404, 2017.

[13] P. Kennedy, A Guide to Econometrics, Blackwell Publishing, Malden, Massachussets, 6 edition, 2008.

[14] A. Pooran, H. Booth, R. F. Miller et al., "Different screening strategies (single or dual) for the diagnosis of suspected latent tuberculosis: a cost effectiveness analysis," BMC Pulmonary Medicine, vol. 10, article no. 7, 2010.

[15] C. R. Horsburgh Jr., "Priorities for the treatment of latent tuberculosis infection in the United States," New England Journal of Medicine, vol. 350, no. 20, pp. 2060-2115, 2004.

[16] Centers for Disease Control and Prevention, "Targeted tuberculin testing and treatment of latent tuberculosis infection. American Thoracic Society," MMWR Recomm Rep. 2000; 49(RR-6):1-51, American Thoracic Society, 2000.

[17] Institute of medicine, Ending Neglect: The Elimination of Tuberculosis in the United States, Institute of Medicine, Washington DC, USA, 2000.

[18] Centers for Disease Control and Prevention, "Updated Guidelines for Using Interferon Gamma Release Assays to Detect Mycobacterium tuberculosis Infection: United States," MMWR Recomm Rep. 2010;59(RR-5):1-26, 2010.

[19] R. Diel, N. Lampenius, and A. Nienhaus, "Cost effectiveness of preventive treatment for tuberculosis in special high-risk populations," PharmacoEconomics, vol. 33, no. 8, pp. 783-809, 2015.

[20] S. Skinner, "Guide to thoracic imaging," Australian Family Physician, vol. 44, no. 8, pp. 558-562, 2015.

[21] United States Census Bureau, "Annual Estimates of the Resident Population for Selected Age Groups by Sex for the United States, States, Counties, and Puerto Rico Commonwealth and Municipios," 2014, https://factfinder.census.gov/faces/ tableservices/jsf/pages/productview.xhtml? src=bkmk.

[22] LG. Hansen and S. Chang, Health research data for the real world: the MarketScan databases, Truven Health Analytics, 2011, White Paper. 
[23] T. Henderson, J. Shepheard, and V. Sundararajan, "Quality of diagnosis and procedure coding in ICD-10 administrative data," Medical Care, vol. 44, no. 11, pp. 1011-1019, 2006.

[24] J. W. Peabody, J. Luck, S. Jain, D. Bertenthal, and P. Glassman, "Assessing the accuracy of administrative data in health information systems," Medical Care, vol. 42, no. 11, pp. 1066-1072, 2004.

[25] R. Trivedi, S. Saksena, and R. K. Gupta, "Magnetic resonance imaging in central nervous system tuberculosis," Indian Journal of Radiology and Imaging, vol. 19, no. 4, pp. 256-265, 2009.

[26] K. Owusu-Edusei, E. L. Stockbridge, C. A. Winston, M. Kolasa, and R. Miramontes, "Tuberculin skin test and interferongamma release assay use among privately insured persons in the United States," The International Journal of Tuberculosis and Lung Disease, vol. 21, no. 6, pp. 684-689, 2017. 


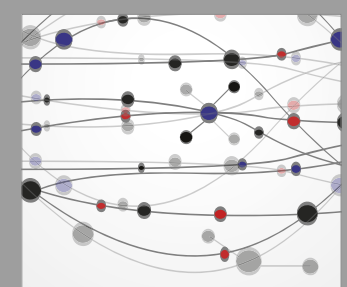

The Scientific World Journal
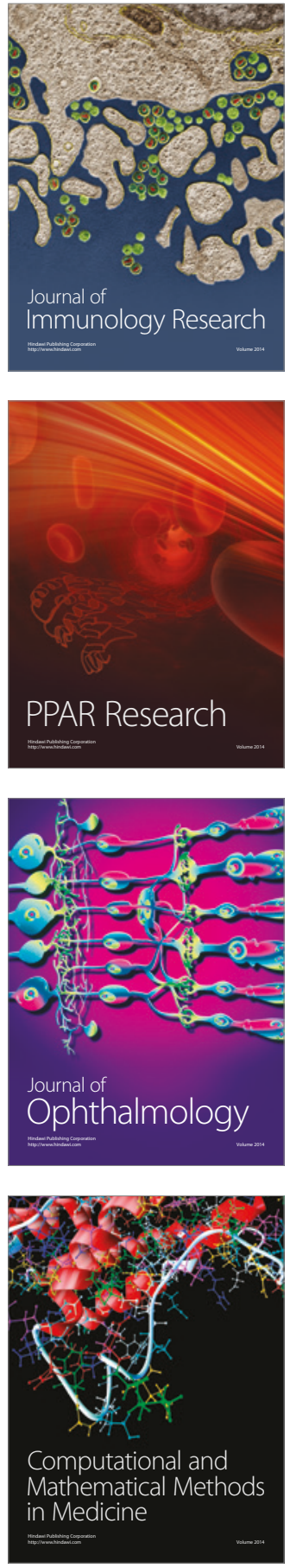

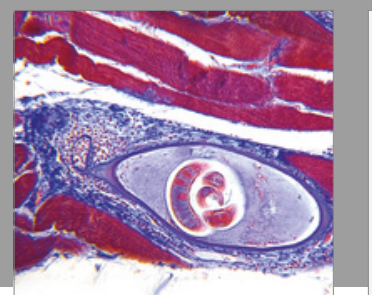

Gastroenterology Research and Practice
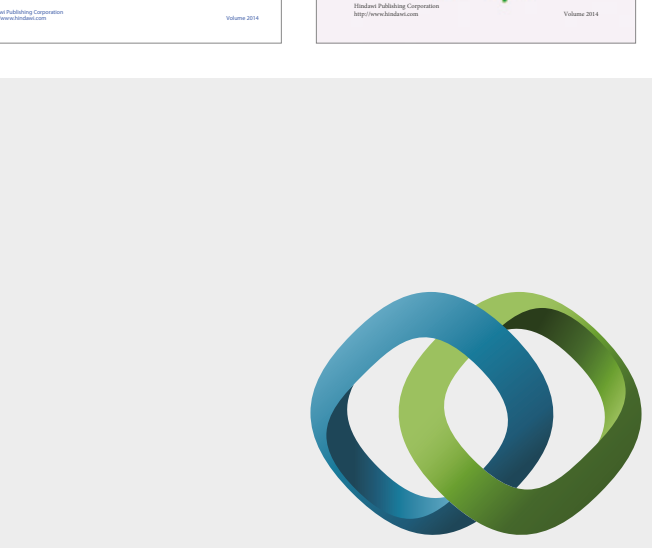

\section{Hindawi}

Submit your manuscripts at

https://www.hindawi.com
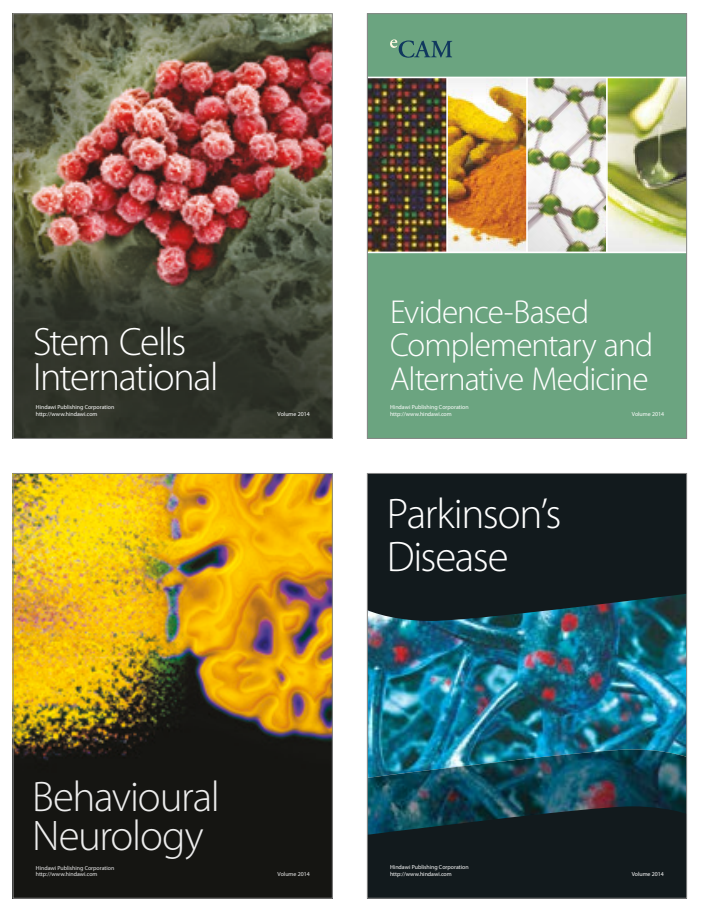
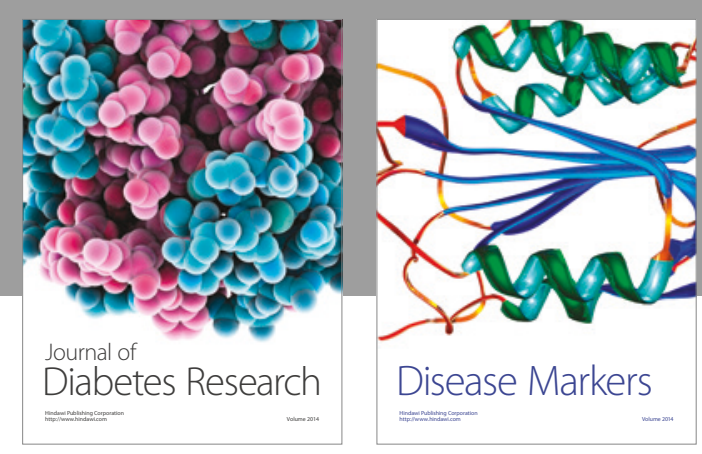

Disease Markers
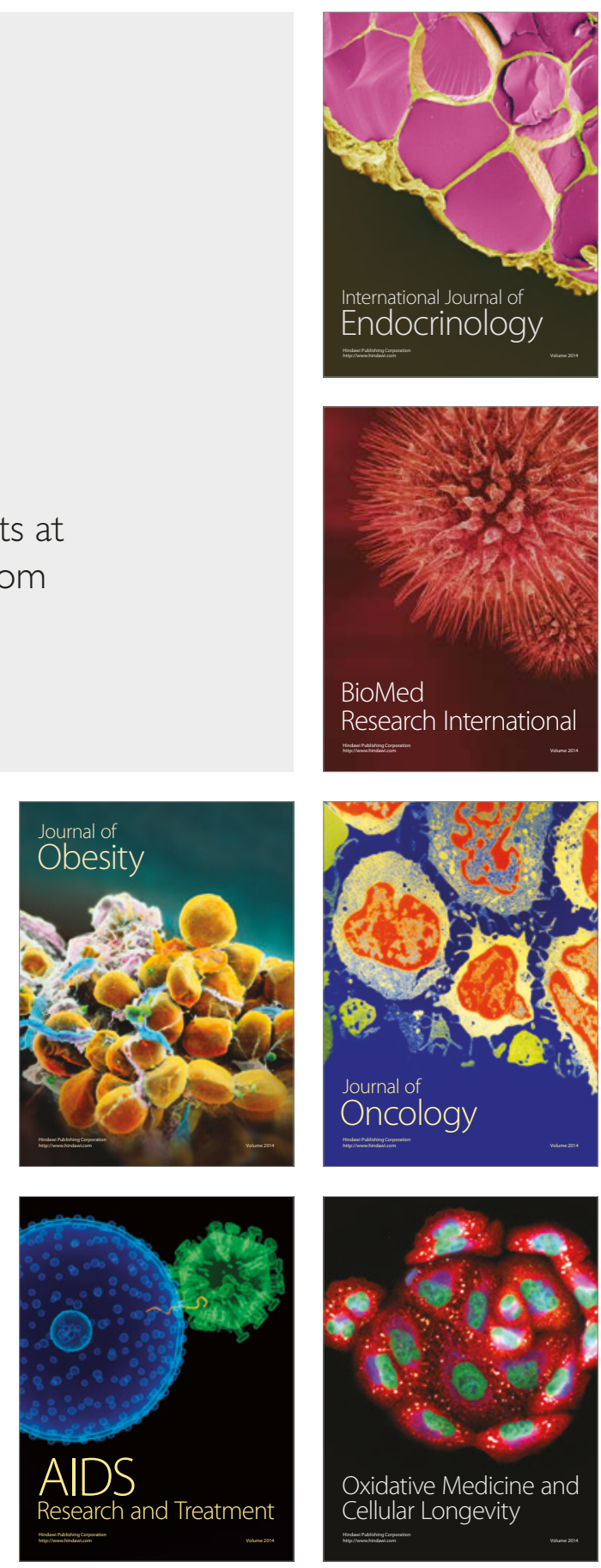